\title{
Estado nutricional de crianças assistidas em creches e situação de (in)segurança alimentar de suas famílias
}

\author{
Nutritional status of children attended in day-care-centers \\ and food (in)security of their families
}

Maercio Mota de Souza ${ }^{1}$

Dixis Figueroa Pedraza ${ }^{2}$

Tarciana Nobre de Menezes ${ }^{3}$
${ }^{1}$ Programa de PósGraduação em Saúde Pública, Universidade Estadual da Paraíba. Travessa Almirante Alexandrino 58, Centro. 58400-265 Campina Grande PB.

maerciomota@bol.com.br

\begin{abstract}
The scope of this study was to examine associated factors with overweight, stunting and underweight in children attending state day care centers of João Pessoa, as well as to describe the situation of food (in) security of their families. A cross-sectional study was conducted with a sample of 250 children. Socioeconomic, maternal and child variables were studied. The nutritional status of children was evaluated considering the height-for-age and weight-for-height indices. Household food security was assessed using the Brazilian Scale of Food Insecurity. The proportions of stunting, underweight and overweight were $7.6 \%, 1.6 \%$ and $6.4 \%$ respectively. The associated factors with short stature were low maternal stature and incomplete vaccination. In relation to low weight, the associated factor was maternal age under 20 years. The overweight child was associated with excessive maternal weight and maternal short stature. In $59.6 \%$ of families, there was food and nutrition insecurity, the mild form being more frequent (32.4\%). Overweight and stunting were found to be the most common nutritional disorders in this study, constituting priorities that should be considered in current public policies.
\end{abstract}

Key words Child malnutrition, Anthropometrics, Preschool
Resumo Examinar fatores associados a excesso de peso, déficit de estatura e déficit de peso em crianças assistidas em creches estaduais de João Pessoa e descrever a situação de (in) segurança alimentar das suas famílias. Trata-se de um estudo transversal com amostra de 250 crianças. Foram estudadas variáveis socioeconômicas, maternas e das crianças. O estado nutricional das crianças foi avaliado considerando os indices estatura-para-idade e peso-para-estatura. A segurança alimentar familiar foi avaliada com a utilização da Escala Brasileira de Insegurança Alimentar. As proporções de déficit de estatura, déficit de peso e excesso de peso foram $7,6 \%, 1,6 \%$ e 6,4\%, respectivamente. Os fatores associados à baixa estatura foram baixa estatura materna e esquema vacinal incompleto. Com relação ao baixo peso, o fator associado foi idade materna inferior a 20 anos. O excesso de peso infantil apresentou associação com o excesso de peso materno e baixa estatura materna. Em 59,6\% das famílias, observouse situação de insegurança alimentar e nutricional, sendo mais frequente a forma leve $(32,4 \%)$. Excesso de peso e déficit de estatura foram os distúrbios nutricionais mais frequentes neste estudo, constituindo prioridades que devem ser consideradas nas políticas públicas atuais.

Palavras-chave Desnutrição infantil, Antropometria, Pré-escolar 


\section{Introdução}

A desnutrição na infância constitui um dos maiores problemas de saúde pública enfrentados pelos países em desenvolvimento, devido à sua alta prevalência e aos danos causados ao organismo humano ${ }^{1}$. A definição operacional da desnutrição é, em geral, realizada com o uso de um ou mais índices que calculam a posição relativa das medidas antropométricas na distribuição de valores de referência; em seguida, pela aplicação de um ponto de corte, classifica-se o estado nutricional do indivíduo em saudável ou não. Esses índices são conhecidos como estatura-para-idade (E/ $\mathrm{I})$, peso-para-idade $(\mathrm{P} / \mathrm{I})$ e peso-para-estatura $(\mathrm{P} / \mathrm{E})^{2}$.

$\mathrm{O}$ índice $\mathrm{E} / \mathrm{I}$ é um indicador que sofre pouca variação em curtos intervalos de tempo. O comprometimento da altura da criança revela desnutrição de longa duração (formas crônicas), encontrando-se frequentemente associado a condições econômicas gerais de pobreza, infecções repetidas e ingestão inadequada de nutrientes. $\mathrm{O}$ índice $\mathrm{P} / \mathrm{E}$, quando comprometido, reflete uma perda de peso atual, podendo ser interpretado como indicativo de processo de desnutrição aguda ou atual ${ }^{3}$.

Segundo Romani e Lira ${ }^{4}$, o retardo estatural constitui, atualmente, a característica antropométrica mais representativa do quadro epidemiológico do crescimento de crianças no Brasil e no mundo. A condição de déficit estatural se estabelece durante os dois primeiros anos de vida ${ }^{5}$. Nos países em desenvolvimento, em geral, o retardo de crescimento inicia-se entre o quarto e o sexto mês de vida, quando ocorre a substituição do aleitamento materno por alimentos de baixo valor nutricional e frequentemente contaminados, com consequente vulnerabilidade para infecções, especialmente as diarreicas. Além disso, a baixa condição socioeconômica figura como outro importante fator associado à ocorrência deste retardo estatural na infância ${ }^{4}$. Com relação à nutrição infantil, as deficiências de micronutrientes são consideradas fatores de risco potenciais para o déficit de crescimento linear em crianças, sendo o zinco, a vitamina A e o ferro os de maior impacto sobre o problema. Considera-se que as deficiências de ferro e vitamina A afetam o crescimento somente em condições de deficiência severa $(\mathrm{Hb}<9,5 \mathrm{~g} / \mathrm{dL}$, retinol sérico $<0,35$ $\mu \mathrm{mol} / \mathrm{L})$. O contrário é observado na deficiência de zinco (zinco sérico $<65 \mathrm{mg} / \mathrm{dL}$ ), considerada a de maior importância no crescimento e, portanto, a causa mais comum do déficit de estatura em crianças, tanto nos casos de deficiência severa, como leve ou moderada ${ }^{6,7}$.

Outros fatores, além dos processos infecciosos, da situação socioeconômica e do estado nutricional de micronutrientes, apresentam associação importante à desnutrição infantil, como o peso e o comprimento ao nascer, a escolaridade materna, a ocupação da mãe, a estatura materna e as condições de saneamento ambiental ${ }^{4}$.

A desnutrição crônica no Brasil teve uma redução de, aproximadamente, 50\% nos últimos 10 anos, saindo de um patamar de 13,0 para 7,0\% (ao se comparar os valores da Pesquisa Nacional Sobre Demografia e Saúde da criança e da mulher, PNDS, dos anos 1996 e 2006). Os dados de 2006 indicam que a prevalência do déficit de estatura varia notavelmente nas regiões brasileiras, situando-se entre valores próximos da média nas regiões Sul e Sudeste, e 15,0\% na região Norte. A maior redução da desnutrição crônica, comparando com os valores de 1996, foi encontrada na região Nordeste (de 22,1\% para 5,9\%), seguida pela região Centro-Oeste $(11,0 \% \text { para } 6,0 \%)^{8}$.

Ao mesmo tempo em que se percebe a redução contínua dos casos de desnutrição, são observadas prevalências crescentes de sobrepeso e obesidade. O aumento da prevalência da obesidade no Brasil é proporcionalmente mais elevado nas famílias de baixa renda, podendo coexistir, no mesmo domicílio, indivíduos obesos e desnutridos, caracterizando o processo de transição nutricional ${ }^{9}$.

Além disto, inquéritos nacionais sobre saúde e nutrição mostram que, apesar da redução contínua dos casos de desnutrição, existem importantes diferenças regionais marcadas nas populações infantis mais pobres, residentes nas áreas rurais ou na periferia das cidades mais ricas, as mais sujeitas às carências e suas consequências. A nutrição adequada de tais segmentos continua sendo um dos mais amplos desafios para as políticas públicas no Brasil ${ }^{10,11}$.

Segundo Silva et al. $^{5}$, programas que oferecem cuidados infantis de forma integral e com boa qualidade, destinados às crianças, desde os primeiros anos de vida, podem exercer papel preventivo no sentido de as proteger, sobretudo, de desnutrição crônica, bem como de outros distúrbios nutricionais. Ressalta-se, também, a importância do acesso à assistência à saúde, condições de infraestrutura domiciliar e orientações às mães visando à ampliação do período de aleitamento materno e melhora nos cuidados infantis.

Estudos têm demonstrado associação positiva entre a permanência de crianças em creches e 
seu estado nutricional, constatando-se a redução dos déficits de peso e estatura, principalmente, entre as crianças com maior tempo de permanência. Entretanto, há também fatores negativos relacionados à frequência de crianças às creches como o aumento de episódios de doenças infecto-contagiosas e de outras doenças de maior gravidade, podendo repercutir negativamente no estado nutricional. É importante ressaltar que estes aspectos negativos podem ser minimizados por meio de medidas preventivas específicas e que a utilização das creches por crianças em condições socioeconômicas menos favorecidas pode ser considerada uma das estratégias dos países em desenvolvimento para garantir o crescimento e o desenvolvimento das mesmas ${ }^{12}$.

Embora a fome e a desnutrição sejam as manifestações mais cruéis da situação de insegurança alimentar e a incapacidade de acesso aos alimentos a sua principal causa, outros aspectos devem também ser considerados ${ }^{13}$, tais como obesidade, doenças associadas à má alimentação e consumo de alimentos de qualidade duvidosa ou prejudicial à saúde ${ }^{14}$.

De acordo com Pimentel et al. ${ }^{15}$, há escassez na literatura científica de estudos relacionando a segurança alimentar (mensurada pela Escala Brasileira de Insegurança Alimentar - EBIA) e indicadores do estado nutricional de crianças.

É objetivo do presente estudo examinar fatores associados a excesso de peso, déficit de estatura e déficit de peso em crianças assistidas em creches de João Pessoa, bem como descrever a situação de (in)segurança alimentar das suas famílias.

\section{Métodos}

Trata-se de um estudo transversal inserido na pesquisa "Estado nutricional, consumo de alimentos e segurança alimentar de crianças assistidas em creches do Programa Vida Criança de João Pessoa". O estudo foi desenvolvido em creches localizadas em João Pessoa, sob administração da Secretaria de Desenvolvimento Humano do Governo do Estado da Paraíba. O município de João Pessoa, capital do estado da Paraíba, dispõe de 30 creches públicas estaduais que assistem uma população de, aproximadamente, 2800 crianças em idade pré-escolar.

Utilizou-se uma amostragem probabilística estimada das crianças usuárias de creches, conglomerada em dois estágios de seleção (creches e crianças). Para determinar o tamanho amostral partiu-se de uma prevalência de 7\% de déficit de estatura, segundo a PNDS, $2006^{8}$, de forma que uma amostra composta por, no mínimo, 252 crianças asseguraria a obtenção de estimativas para proporções sob níveis de precisão máximos iguais a $3 \%$, que corrigido em $5 \%$, para compensar eventuais perdas inerentes ao trabalho, perfaria o total de 265 crianças a serem investigadas. No primeiro estágio, 11 creches foram determinadas por meio de seleção sistemática com probabilidades proporcionais ao tamanho da população infantil assistida por cada creche, do total das 30 creches, garantindo a representatividade para as variáveis a serem estudadas. No estágio seguinte, em cada creche selecionada foram aleatorizadas crianças em número proporcional ao número total de assistidas por cada creche com equiprobabilidade dentre o total de crianças da creche.

A coleta de dados foi realizada nas creches, no período de 12 de maio de 2008 a 19 de março de 2009. Para esses fins foi formada uma equipe de entrevistadores devidamente treinados pelo coordenador da pesquisa. O treinamento incluiu o preenchimento de um questionário, assim como as orientações sobre as técnicas para a aferição do peso e da estatura das crianças e das mães. O questionário, aplicado às mães ou aos responsáveis pelas crianças, continha informações sobre condições socioeconômicas da família (a partir de dados secundários), características maternas e características das crianças.

As variáveis de estudo foram:

1. Variáveis dependentes: índices P/E e E/I das crianças.

2. Variáveis independentes:

- condições socioeconômicas: renda familiar, tipo de casa, número de cômodos no domicílio, número de indivíduos no domicílio;

- características maternas: cuidados durante a gravidez (orientação sobre amamentação, suplementação com sulfato ferroso, imunização antitetânica, realização de três ou mais consultas de pré-natal, não ter feito uso de cigarro nem de álcool durante o período gestacional), idade, estatura-para-idade, Índice de Massa Corporal (IMC).

- características das crianças: sexo, idade, esquema vacinal, peso ao nascer;

Nas crianças, foram realizadas aferições de peso, de comprimento (menores de 24 meses) e de estatura (maiores de 24 meses), seguindo os procedimentos recomendados pela Organização Mundial da Saúde (OMS) ${ }^{16}$. Para aferir o peso, foi utilizada balança digital da marca Tanita UM-080 ${ }^{\circledR}$, com variação de 100 gramas. Antes 
da pesagem de crianças menores de 24 meses, foram retiradas roupas e acessórios (fralda, chupeta, chuquinhas, entre outros) e a aferição do peso foi feita pela diferença entre o valor obtido da pesagem conjunta da mãe com a criança em seu colo e o valor do peso da mãe. O comprimento foi aferido com auxílio de um infantômetro de madeira portátil com amplitude de 130 $\mathrm{cm}$ e subdivisões de $0,1 \mathrm{~cm}$, estando a criança deitada em um colchão próprio para aferição, com a cabeça posicionada próxima à prancha imóvel, mantendo-a paralela à régua, com os joelhos pressionados, e os pés juntos com tornozelos em ângulo de $90^{\circ}$, mantidos pela prancha móvel, fazendo-se assim a leitura. A estatura foi aferida utilizando o estadiômetro WCS com escala em milímetros ( $\mathrm{mm}$ ) na posição vertical, com a criança posicionada ereta, com os pés juntos e os braços estendidos ao longo do corpo, acertando a cabeça de forma que o plano de Frankfort ficasse paralelo ao chão. O comprimento/estatura foi aferido em duplicata.

A avaliação do estado nutricional infantil foi realizada segundo os índices antropométricos $\mathrm{P} /$ E e E/I, de acordo com sexo e faixa etária, utilizando como referência a distribuição recomendada pela OMS em $2004^{17}$. Foram enquadradas na categoria com déficit de peso e com déficit de estatura crianças cujos índices P/E e E/I, respectivamente, foram inferiores a $-2,0$ escores $\mathrm{z}$. Foram consideradas com excesso de peso as crianças cujo índice P/ $\mathrm{E}$ foi igual ou superior a $+2,0$ escores $\mathrm{z}$.

A baixa estatura materna foi definida pelo ponto de corte $155,0 \mathrm{~cm}^{18,19}$. O IMC da mãe também foi utilizado para a classificação do estado nutricional materno, empregando os pontos de corte indicados pela OMS (1995) ${ }^{17}$. Os instrumentos utilizados para a mensuração do peso e da estatura das mães, assim como os procedimentos de medição, foram os mesmos referidos para as crianças.

Para avaliar a associação das condições socioeconômicas, das características maternas e das características das crianças com o estado nutricional das crianças, atendeu-se às especificações dos modelos hierarquizados, utilizando o modelo em três níveis de determinação: distal/condições socioeconômicas (renda familiar, tipo de casa, número de cômodos no domicílio, número de indivíduos no domicílio), intermediário/características maternas (cuidados na gravidez, idade da mãe, estatura-para-idade da mãe, Índice de Massa Corporal da mãe), e proximal/características das crianças (sexo da criança, idade da criança, esquema vacinal, peso ao nascer $)^{20}$.
A situação de segurança alimentar das famílias das crianças foi avaliada com a aplicação da Escala Brasileira de Insegurança Alimentar (EBIA). O instrumento validado é composto por 15 perguntas que possibilitam a avaliação da segurança alimentar intrafamiliar por meio da percepção do entrevistado, responsável pela criança, associada à certeza de garantir a alimentação e a reação dos lares ante essa percepção. As questões incluem a preocupação e a ansiedade da família em relação à obtenção dos alimentos, perpassando pelo comprometimento da qualidade e quantidade da dieta de adultos e crianças, alcançando situações mais graves, com restrições quantitativas de alimentos para todos os membros da família ${ }^{21,22}$.

A EBIA possibilita determinar os níveis de segurança alimentar familiar, por meio da quantificação do total de respostas afirmativas na escala:

- Segurança Alimentar: 0 resposta positiva;

- Insegurança Alimentar Leve: 1 - 5 respostas positivas;

- Insegurança Alimentar Moderada: 6 - 10 respostas positivas;

- Insegurança Alimentar Grave: 11 - 15 respostas positivas.

A digitação dos dados foi realizada com dupla entrada independente em planilhas do programa Excel (Microsoft Inc., Estados Unidos), de maneira tal que possibilitou a unificação entre os mesmos, por meio de uma única variável identificadora da criança. Após o término da digitação, os dois bancos de dados foram cruzados com a utilização do aplicativo Validate do programa Epi Info v. 6.04b (WHO/CDC, Atlanta, Estados Unidos), possibilitando assim verificar a consistência dos dados e gerando o banco final que foi usado para análise estatística.

Para a análise estatística foi utilizada a regressão logística com abordagem das análises bivariada e multivariada. Foram consideradas elegíveis para as análises multivariadas as variáveis explicativas que obtiveram $\mathrm{p}$-valor $<0,25$ na análise bivariada ${ }^{23}$. Para a regressão multivariada ${ }^{18}$ foi utilizado o método forward e as demais conclusões foram tomadas ao nível de significância de $10 \%$. No modelo hierarquizado foram rodados vários modelos multivariados de regressão logística, iniciando-se com as variáveis explicativas pertencentes ao grupo mais distal, no qual eram introduzidas uma a uma (método forward), permanecendo as variáveis estatisticamente associadas ao desfecho (índices antropométricos da criança), ao nível de significância de 10\%. Repetiu-se o mesmo passo para o nível subsequen- 
te (intermediário e proximal). Ao analisar as variáveis do nível intermediário, permaneceram no modelo as variáveis do nível distal, mesmo ocorrendo alteração de significância estatística das variáveis. Foi considerado o nível de significância de 5\% para indicar associação. Os softwares utilizados foram o Excel 2000 e o R v2.10.0.

Todas as diretrizes éticas do Conselho Nacional de Saúde, bem como os princípios éticos referentes às publicações de pesquisa com seres humanos contidos na Declaração de Helsinki foram contempladas e o projeto maior foi aprovado pelo Comitê de Ética em Pesquisa da Universidade Estadual da Paraíba.

\section{Resultados}

Do total de 265 crianças elegíveis para o estudo, 15 foram perdidas pela ausência à creche no momento da coleta de dados, implicando uma amostra final de 250 crianças estudadas.

A avaliação do estado nutricional da amostra revelou que 7,6\% (IC95\% 4,5\% - 11,1\%) das crianças apresentaram déficit de estatura, ao passo que apenas $1,6 \%$ (IC95\% 0,2\% - 3,7\%) apresentaram déficit de peso. $\mathrm{O}$ excesso de peso foi frequente em 6,4\% (IC95\% 3,0\% - 8,9\%) das crianças.

Nas Tabelas 1 e 2 encontram-se os fatores preditivos de déficit de estatura infantil submetidos à análise bivariada e multivariada hierarquizada, respectivamente. Após análise bivariada, constatou-se que a baixa estatura da mãe ( $p=$ 0,0241 ; OR $=3,36$; IC95\% 1,24-10,68) se associou significantemente à ocorrência de déficit de estatura infantil. Ressalta-se que o esquema vacinal incompleto mostrou-se mais fortemente associado à baixa estatura infantil $(p=0,0049$; $\mathrm{OR}=4,62$; IC95\% 1,49 - 13,05) quando comparado aos demais fatores preditivos.

O modelo multinível hierarquizado confirma a associação entre o déficit de estatura infantil e as mesmas variáveis associadas na análise bivariada. Entretanto, o déficit de estatura materno $(p=0,0197$; OR = 3,96; IC95\% 1,31 - 13,79) apresentou maior força de associação para a ocorrência do desfecho, quando comparada ao esquema vacinal incompleto $(p=0,0319 ; \mathrm{OR}=$ 3,62; IC95\% 1,06-11,50).

A análise bivariada dos fatores associados ao déficit de peso infantil evidencia que a idade materna inferior a 20 anos representou um fator de associação altamente significante ( $p=0,0158$; OR $=11,97$; IC $95 \% 1,37-10,23)$. Os demais fatores não mostraram associação. No modelo hierarquizado, o mesmo fator permaneceu associado significantemente ao baixo peso ( $p=0,0158$; OR $=11,97$; IC95\% 1,37-10,23).

A Tabela 3 mostra as variáveis associadas ao excesso de peso infantil na análise bivariada. Dentre as variáveis do nível intermediário (características maternas), o excesso de peso materno, representado pelo índice de massa corporal (IMC) acima de $25 \mathrm{Kg} / \mathrm{m}^{2}$, apresentou associação significante $(p=0,0128$; OR $=0,23$; IC95\% $0,06-0,68)$ ao desfecho excesso de peso infantil.

Os fatores associados ao excesso de peso infantil, após o modelo hierarquizado exposto na Tabela 4, foram o excesso de peso materno ( $p=$ 0,$0155 ;$ OR $=0,23$; IC95\% 0,06-0,71) e a baixa estatura materna ( $p=0,0554$; OR $=0,31$; IC95\% $0,08-0,95)$.

A situação de segurança alimentar das famílias das crianças avaliadas revelou que $40,4 \%$ das famílias foram classificadas na categoria de segurança alimentar, predominando, portanto, a condição de insegurança alimentar $(59,6 \%)$, sendo mais frequente a forma leve $(32,4 \%)$, seguida pela forma moderada $(18,0 \%)$ e, por último, pela forma grave (9,2\%) (Gráfico 1). No Gráfico 1, nota-se que nas crianças com estatura e peso normal houve maior proporção de segurança alimentar, e que nas crianças com déficit de estatura a proporção mais expressiva foi a de insegurança alimentar leve (42,0\%). Ressalta-se, também, a alta prevalência de insegurança alimentar leve $(43,8 \%)$ nas crianças com excesso de peso, próximo do valor de 50,0\% de segurança alimentar nesse grupo de crianças.

\section{Discussão}

O estudo dos fatores determinantes da ocorrência de qualquer enfermidade requer a adoção de um modelo teórico que relacione e articule os fatores potenciais que contribuem para sua determinação. O modelo de determinação dos distúrbios nutricionais na infância adotado neste trabalho postula que o déficit de estatura, o déficit de peso e o excesso de peso nas crianças estudadas se associam a fatores de diferentes níveis de complexidade, a exemplo das condições socioeconômicas da família, das características maternas e das características da própria criança. Nesse sentido, as condições sociais e econômicas se comportam como importantes preditores do estado nutricional infantil, interferindo imediatamente sobre as características da mãe, representadas pelo seu es- 
Tabela 1. Distribuição do déficit de estatura das crianças de acordo com as variáveis socioeconômicas, características maternas e características das crianças e os respectivos OR, intervalo de confiança 95\% e pvalor, João Pessoa - PB, 2008 - 2009.

\begin{tabular}{|c|c|c|c|c|c|c|c|}
\hline \multirow{2}{*}{ Variáveis } & \multicolumn{2}{|c|}{ Total } & \multicolumn{4}{|c|}{ Déficit de estatura } & \multirow{2}{*}{$\begin{array}{c}\text { p- } \\
\text { valor }\end{array}$} \\
\hline & $\mathbf{N}$ & $\%$ & $\mathbf{n}$ & $\%$ & OR & IC 95\% & \\
\hline \multirow{2}{*}{\multicolumn{8}{|c|}{$\begin{array}{l}\text { Nível distal: Condições socioeconômicas } \\
\text { Renda familiar* }\end{array}$}} \\
\hline & & & & & & & \\
\hline$\geq 1 / 2 \mathrm{SM}$ & 195 & 78,0 & 13 & 6,7 & 1,00 & & \\
\hline$<1 / 2 \mathrm{SM}$ & 55 & 22,0 & 6 & 10,9 & 1,71 & $0,58-4,58$ & 0,30 \\
\hline \multicolumn{8}{|l|}{ Tipo de casa } \\
\hline Própria/alugada & 215 & 86,0 & 16 & 7,4 & 1,00 & & \\
\hline Invadida/cedida & 35 & 14,0 & 3 & 8,6 & 1,17 & $0,26-3,75$ & 0,81 \\
\hline \multicolumn{8}{|c|}{ Número de cômodos no domicílio } \\
\hline$\geq 4$ & 198 & 79,2 & 12 & 6,1 & 1,00 & & \\
\hline$<4$ & 52 & 20,8 & 7 & 13,5 & 2,41 & $0,86-6,35$ & 0,08 \\
\hline \multicolumn{8}{|c|}{ Número de indivíduos no domicílio } \\
\hline$<6$ & 180 & 72,0 & 12 & 6,7 & 1,00 & & \\
\hline$\geq 6$ & 70 & 28,0 & 7 & 10,0 & 1,56 & $0,56-4,05$ & 0,37 \\
\hline \multirow{2}{*}{\multicolumn{8}{|c|}{$\begin{array}{l}\text { Nível intermediário: Características maternas } \\
\text { Cuidados na gravidez }\end{array}$}} \\
\hline & & & & & & & \\
\hline Bons & 155 & 62,0 & 11 & 7,1 & 1,00 & & \\
\hline Ruins & 95 & 38,0 & 8 & 8,4 & 1,20 & $0,45-3,09$ & 0,70 \\
\hline \multicolumn{8}{|l|}{ Idade } \\
\hline$\geq 20$ & 229 & 91,6 & 18 & 7,9 & 1,00 & & \\
\hline$<20$ & 21 & 8,4 & 1 & 4,8 & 0,59 & $0,03-3,08$ & 0,61 \\
\hline \multicolumn{8}{|l|}{ Estatura para idade } \\
\hline Estatura normal & 131 & 52,4 & 5 & 3,8 & 1,00 & & \\
\hline Baixa estatura & 119 & 47,6 & 14 & 11,8 & 3,36 & $1,24-10,68$ & 0,02 \\
\hline \multicolumn{8}{|l|}{ Índice de Massa Corporal } \\
\hline Normal & 96 & 40,2 & 4 & 4,2 & 1,00 & & \\
\hline Baixo peso & 16 & 6,7 & 2 & 12,5 & 3,29 & $0,43-18,56$ & 0,19 \\
\hline Excesso de peso & 127 & 53,1 & 11 & 8,7 & 2,18 & $0,72-8,07$ & 0,19 \\
\hline \multicolumn{8}{|c|}{ Nível proximal: Características das crianças } \\
\hline \multicolumn{8}{|l|}{ Sexo } \\
\hline Masculino & 111 & 44,4 & 9 & 8,1 & 1,00 & & \\
\hline Feminino & 139 & 55,6 & 10 & 7,2 & 0,88 & $0,34-2,29$ & 0,79 \\
\hline \multicolumn{8}{|l|}{ Idade } \\
\hline $37-60$ & 165 & 66,0 & 9 & 5,4 & 1,00 & & \\
\hline $6-36$ & 85 & 34,0 & 10 & 11,8 & 2,31 & $0,90-6,05$ & 0,08 \\
\hline \multicolumn{8}{|l|}{ Esquema vacinal } \\
\hline Completo & 223 & 89,2 & 13 & 5,8 & 1,00 & & \\
\hline Incompleto & 27 & 10,8 & 6 & 22,2 & 4,62 & $1,49-13,05$ & 0,005 \\
\hline \multicolumn{8}{|l|}{ Peso ao nascer } \\
\hline Peso normal & 207 & 82,8 & 13 & 6,3 & 1,00 & & \\
\hline Baixo peso ao nascer & 43 & 17,2 & 6 & 13,9 & 2,42 & $0,81-655$ & 0,09 \\
\hline
\end{tabular}

OR: Odds Ratio; IC: Intervalo de Confiança; SM: Salário Mínimo. * Considerando o valor do salário mínimo da época (R $\$ 416,00)$. ** Foram consideradas mães com cuidados bons durante a gravidez aquelas que receberam orientação sobre a amamentação, suplementação com sulfato ferroso, vacina antitetânica, três ou mais consultas pré-natais e que não fizeram uso de cigarro nem de álcool durante o período gestacional.

tado nutricional, pela sua idade, bem como as condições da gravidez. Por sua vez, as características maternas repercutem sobre condições relacionadas com a criança. Os determinantes deste último nível se expressam pela ocorrência de bai- xo peso ao nascer, completitude do esquema vacinal e outros fatores que afetam diretamente o estado nutricional da criança.

À medida que esses determinantes se manifestam como promotores da iniquidade social, 
Tabela 2. Modelo final hierarquizado com as variáveis determinantes dos fatores de risco para as crianças com déficit de estatura e os respectivos OR ajustados, intervalo de confiança 95\% e p-valor, João Pessoa PB, $2008-2009$.

\begin{tabular}{lccc}
\hline \multicolumn{1}{c}{ Variáveis } & OR Ajustado & IC $\mathbf{9 5 \%}$ & p-valor \\
\hline $\begin{array}{l}\text { Número de cômodos no domicílio } \\
\quad \text { < }\end{array}$ & 1,73 & $0,50-5,49$ & 0,36 \\
$\quad \begin{array}{l}\text { Estatura para idade da mãe } \\
\quad \text { Baixa estatura }\end{array}$ & 3,96 & $1,31-13,79$ & $\mathbf{0 , 0 2}$ \\
$\quad \begin{array}{l}\text { Indice de Massa Corporal da mãe } \\
\quad \text { Baixo peso }\end{array}$ & 4,35 & $0,51-28,71$ & 0,14 \\
$\quad$ Excesso de peso & 3,07 & $0,93-12,35$ & 0,08 \\
$\begin{array}{l}\text { Idade da criança } \\
\quad \text { - 36 }\end{array}$ & 2,71 & $0,91-8,39$ & 0,07 \\
$\quad$ Esquema vacinal & & & \\
$\quad$ Incompleto & 3,62 & $1,06-11,50$ & $\mathbf{0 , 0 3}$ \\
\hline
\end{tabular}

OR: Odds Ratio; IC: Intervalo de Confiança.

econômica e da saúde, desencadeiam o comprometimento do estado nutricional infantil, seja na ocorrência de distúrbios carenciais, seja na de excesso de peso. A limitação imposta por esses níveis de determinação restringe a adoção, por parte das mães, por exemplo, de um conjunto de ações que poderiam contribuir na promoção da saúde e nutrição da criança, assim como a restrição da própria criança em superar com êxito as condições adversas do ambiente social e econômico em que está inserida.

Dentre os distúrbios nutricionais apresentados pelas crianças que compuseram este estudo, o déficit de estatura foi o que apresentou a maior frequência. Romani e Lira ${ }^{4}$ qualificam o déficit de crescimento infantil como a característica antropométrica mais representativa do quadro epidemiológico do Brasil. Dados de baixa estatura similares foram encontrados em crianças usuárias de creches em cidades como Florianópolis $(8,7 \%)^{24}$, São Paulo $(7,0 \%)^{25}$ e Piracicaba $(8,2 \%)^{26}$. A frequência de déficit de estatura alcançada no presente estudo exprime sua real magnitude, se considerarmos a classificação adotada pela OMS, em países latinoamericanos, que, a fim de denotar o grau de severidade, enquadra como problema moderado de saúde pública valores de prevalência de déficit de estatura $>5 \%-<25 \%{ }^{18}$.

Segundo de Onis et al. ${ }^{27}$, o crescimento, além de ser o melhor indicador global de bem-estar infantil, é também um excelente indicador de desigualdades nas populações. Permite-se inferir que a forma crônica de desnutrição infantil, representada pela baixa estatura, é um dos produtos da desigualdade social que ainda prevalece no Brasil. Isto pode ser refletido nas maiores prevalências encontradas em populações de maior vulnerabilidade social como crianças menores de 5 anos da macrorregião Norte do país ${ }^{8}(14,8 \%)$, de populações Quilombolas ${ }^{28}(11,6 \%)$ e indígenas $^{29}(15,5 \%)$

A baixa estatura continua, também, a ser um dos problemas importantes de saúde pública, em razão das consequências desastrosas para o crescimento, o desenvolvimento e até para a sobrevivência das crianças ${ }^{11}$. Há fortes evidências de que o retardo no crescimento infantil se associa ao atraso no desenvolvimento mental e se relaciona tanto com desempenho escolar insatisfatório, quanto com a redução da capacidade intelectual, além de ser considerado como um determinante de maior risco para gerar crianças com baixo peso ao nascer ${ }^{11,27,30}$.

A associação entre o estado nutricional materno e o de seus filhos representa um dos principais dilemas da saúde pública contemporânea, centrado no ciclo da desnutrição infantil, déficit de estatura, obesidade e comorbidades na vida adulta, processo iniciado no período intrauterino ${ }^{31}$. A influência exercida pelo estado nutricional da mãe sobre o dos filhos, por compartilharem tanto informações genéticas, quanto condições socioeconômicas e ambientais, apontada no presente estudo, converge com os resultados de outras pesquisas ${ }^{32,33}$.

Estudo realizado por Oliveira et al..$^{34}$ em 15 municípios brasileiros, cuja amostra avaliada foi de 3746 pré-escolares, mostrou forte associação 
Tabela 3. Distribuição do excesso de peso das crianças de acordo com as variáveis socioeconômicas, características maternas e características das crianças e os respectivos OR, intervalo de confiança 95\% e p-valor, João Pessoa - PB, 2008 - 2009.

\begin{tabular}{|c|c|c|c|c|c|c|c|}
\hline \multirow{2}{*}{ Variáveis } & \multicolumn{2}{|c|}{ Total } & \multicolumn{4}{|c|}{ Excesso de peso } & \multirow{2}{*}{$\begin{array}{c}\text { p- } \\
\text { valor }\end{array}$} \\
\hline & $\mathbf{N}$ & $\%$ & $\mathbf{n}$ & $\%$ & OR & IC 95\% & \\
\hline \multicolumn{8}{|c|}{ Nível distal: Condições socioeconômicas } \\
\hline \multicolumn{8}{|c|}{ Renda familiar* } \\
\hline$\geq 1 / 2 \mathrm{SM}$ & 195 & 78,0 & 14 & 7,2 & 1,00 & & \\
\hline$<1 / 2 \mathrm{SM}$ & 55 & 22,0 & 2 & 3,6 & 0,49 & $0,08-1,82$ & 0,35 \\
\hline \multicolumn{8}{|l|}{ Tipo de casa } \\
\hline Própria/alugada & 215 & 86,0 & 15 & 7,0 & 1,00 & & \\
\hline Invadida/cedida & 35 & 14,0 & 1 & 2,8 & 0,39 & $0,02-2,03$ & 0,37 \\
\hline \multicolumn{8}{|c|}{ Número de cômodos no domicílio } \\
\hline$\geq 4$ & 198 & 79,2 & 14 & 7,1 & 1,00 & & \\
\hline$<4$ & 52 & 20,8 & 2 & 3,8 & 0,53 & $0,08-1,96$ & 0,40 \\
\hline \multicolumn{8}{|c|}{ Número de indivíduos no domicílio } \\
\hline$<6$ & 180 & 78,0 & 13 & 7,2 & & & \\
\hline$\geq 6$ & 70 & 28,0 & 3 & 4,3 & 0,57 & $0,16-2,08$ & 0,39 \\
\hline \multicolumn{8}{|c|}{ Nível intermediário: Características maternas } \\
\hline \multicolumn{8}{|c|}{ Cuidados na gravidez ${ }^{* *}$} \\
\hline Bons & 145 & 58,0 & 10 & 7,0 & 1,00 & & \\
\hline Ruins & 95 & 42,0 & 6 & 6,31 & 0,98 & $0,32-2,73$ & 0,96 \\
\hline \multicolumn{8}{|l|}{ Idade } \\
\hline$\geq 20$ & 229 & 91,6 & 14 & 6,1 & 1,00 & & \\
\hline$<20$ & 21 & 8,4 & 2 & 9,5 & 1,62 & $0,24-6,38$ & 0,54 \\
\hline \multicolumn{8}{|l|}{ Estatura para idade } \\
\hline Estatura normal & 131 & 52,4 & 12 & 9,2 & 1,00 & & \\
\hline Baixa estatura & 119 & 47,6 & 4 & 3,4 & 0,34 & $0,09-1,02$ & 0,07 \\
\hline \multicolumn{8}{|l|}{ Índice de Massa Corporal } \\
\hline Normal & 96 & 40,2 & 12 & 12,5 & 1,00 & & \\
\hline Baixo peso & 16 & 6,7 & 0 & 0 & - & - & 0,99 \\
\hline Excesso de peso & 127 & 53,1 & 4 & 3,1 & 0,23 & $0,06-0,68$ & 0,0128 \\
\hline \multicolumn{8}{|c|}{ Nível proximal: Características das crianças } \\
\hline \multicolumn{8}{|l|}{ Sexo } \\
\hline Masculino & 111 & 44,4 & 8 & 7,2 & 1,00 & & \\
\hline Feminino & 139 & 55,6 & 8 & 5,7 & 0,79 & $0,28-2,21$ & 0,64 \\
\hline \multicolumn{8}{|l|}{ Idade } \\
\hline $37-60$ & 165 & 66,0 & 8 & 4,8 & 1,00 & & \\
\hline $6-36$ & 85 & 34,0 & 8 & 9,4 & 2,04 & $0,72-5,74$ & 0,16 \\
\hline \multicolumn{8}{|l|}{ Esquema vacinal } \\
\hline Completo & 223 & 89,2 & 15 & 6,7 & 1,00 & & \\
\hline Incompleto & 27 & 10,8 & 1 & 3,7 & 0,53 & $0,03-2,80$ & 0,55 \\
\hline \multicolumn{8}{|l|}{ Peso ao nascer } \\
\hline Peso normal & 207 & 82,8 & 13 & 6,3 & 1,00 & & \\
\hline Baixo peso ao nascer & 43 & 17,2 & 3 & 7,0 & 1,12 & $0,25-3,67$ & 0,86 \\
\hline
\end{tabular}

OR: Odds Ratio; IC: Intervalo de Confiança; SM: Salário Mínimo. * Considerando o valor do salário mínimo da época (R\$ $416,00)$. ${ }^{* *}$ Foram consideradas mães com cuidados bons durante a gravidez aquelas que receberam orientação sobre a amamentação, suplementação com sulfato ferroso, vacina antitetânica, três ou mais consultas pré-natais e que não fizeram uso de cigarro nem de álcool durante o período gestacional.

entre o esquema vacinal incompleto e a baixa estatura infantil em modelo multinível final, resultado que corrobora a mesma associação observada nesta pesquisa, representando o único determinante proximal de significante associação no modelo final. No modelo causal proposto pela $\mathrm{UNICEF}^{35}$, a desnutrição infantil é resultado de uma dieta inadequada e do acometimento de doenças decorrentes de fatores como cuidados inadequados e serviços de saúde deficientes. 
Tabela 4. Modelo final hierarquizado com as variáveis determinantes dos fatores de risco para as crianças com excesso de peso e os respectivos OR ajustados, intervalo de confiança 95\% e p-valor, João Pessoa - PB, $2008-2009$.

\begin{tabular}{lccc}
\hline \multicolumn{1}{c}{ Variáveis } & OR Ajustado & IC 95\% & p-valor \\
\hline $\begin{array}{l}\text { Estatura para idade da mãe } \\
\text { Baixa estatura } \\
\text { Índice de Massa Corporal da mãe } \\
\text { Excesso de peso }\end{array}$ & 0,31 & $0,08-0,95$ & $\mathbf{0 , 0 5 5 4}$ \\
\hline
\end{tabular}

OR: Odds Ratio; IC: Intervalo de Confiança.
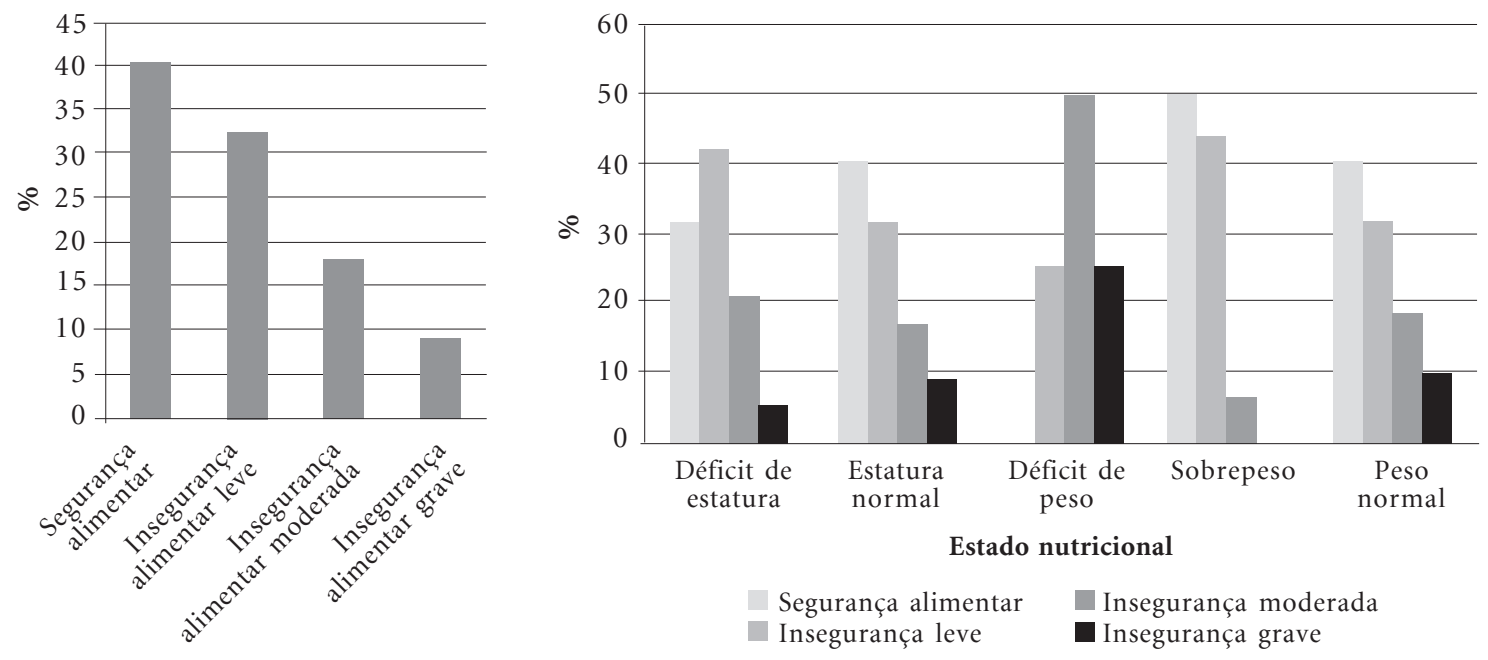

Gráfico 1. Proporção dos níveis de insegurança alimentar familiar geral e por categoria do estado nutricional de crianças institucionalizadas em creches do Programa Vida Criança, João Pessoa - PB, 2008-2009.

Doenças virais e bacterianas podem produzir anorexia e reduzir a ingestão de nutrientes, problemas de absorção e utilização, podendo afetar o crescimento infantil ${ }^{30}$.

O desenvolvimento precoce da obesidade vem apresentando valores alarmantes entre crianças, adolescentes e adultos em todo o mundo, sendo um problema de saúde pública que tende a se manter em todas as fases da vida. O excesso de peso como problema de saúde pública tem suplantado, em muito, o déficit de peso-para-estatura no Brasil e em outros países ${ }^{36}$. A mobilidade social dessa condição constitui a característica epidemiológica mais marcante do processo de transição nutricional da população brasileira ${ }^{9}$.
A proporção de excesso de peso entre crianças usuárias de creches de João Pessoa $(8,4 \%)$ é semelhante às encontradas em estudos realizados com crianças assistidas por creches públicas de cidades como São Paulo ${ }^{37}$ (6,2\%), Brasília ${ }^{38}$ $(6,1 \%)$ e Florianópolis ${ }^{39}(8,6 \%)$. Destaque, também, para a similaridade com a proporção de 7,4\% observada nas crianças brasileiras, segundo resultados da PNDS $^{8}$. Esses resultados são ainda mais preocupantes se consideradas as evidências de que proporção significativa das crianças com excesso de peso/obesidade se torna adultos obesos com alto risco de sofrer outras doenças crônicas não transmissíveis como o diabetes mellitus ${ }^{40-42}$. Sendo assim, a prevenção do exces- 
so de peso/obesidade deve começar desde o nascimento, enfocando que os hábitos alimentares são formados nos primeiros anos de vida ${ }^{43}$.

A associação encontrada no modelo multinível final do estudo entre o excesso de peso infantil e o sobrepeso/obesidade da mãe coincide com achados que apontam correlação intrafamiliar positiva quanto ao estado nutricional de pais e filhos $^{44,45}$. É conhecido o caráter familiar da obesidade, constatado pela concomitância entre o excesso de peso em crianças e nos seus pais ${ }^{46}$. Estudos que investigam o aparecimento da obesidade precocemente dão suporte à importância da influência do ambiente familiar sobre o risco de a criança tornar-se obesa, uma vez que a criança sofre grande dependência da decisão dos pais ou familiares. Atitudes da família em relação à compra e apresentação dos alimentos, os hábitos de alimentação e de atividade física e o suporte oferecido para a promoção de atividades de lazer podem influenciar o padrão de alimentação e de atividade física da criança ${ }^{40,41}$.

A baixa estatura materna também se associou ao excesso de peso infantil na análise multinível deste estudo. Evidências sistematizadas da literatura apontam predominante associação positiva entre o baixo peso ao nascer e o desenvolvimento de algum tipo de obesidade na infância. Assim, a baixa estatura materna pode ser interpretada como fator que predispõe à ocorrência de baixo peso ao nascer, determinante proximal do estado nutricional infantil, condição de risco para a ocorrência de excesso de peso ainda na infância ${ }^{47}$.

A frequência de déficit de peso inferior à encontrada na distribuição de referência, ou seja, abaixo do limiar de $2,3 \%$, como a encontrada no presente estudo, representa um risco virtualmente nulo de desnutrição aguda na população. Essa mesma situação foi relatada ao se encontrar uma prevalência de 1,6\% em dados de abrangência nacional ${ }^{8}$.

A associação significante no modelo multinível final do déficit de peso infantil com a idade materna inferior a 20 anos reforça a necessidade de priorizar a vigilância nutricional às mães adolescentes em função do maior risco nutricional a que seus filhos estão submetidos ${ }^{48}$.

No que diz respeito à situação de segurança alimentar das famílias de crianças usuárias de creches de João Pessoa, a proporção da condição de insegurança alimentar (59,6\%) é superior àquela encontrada pela $\mathrm{PNAD}^{49}$, em 2009, para o estado da Paraíba $(41,0 \%)$. Entretanto, quando comparada à prevalência da região Nordeste, no mesmo ano $(46,1 \%)$, percebe-se uma diferença menor. Esta superioridade sugere que as famílias deste estudo apresentam condições socioeconômicas menos favorecidas do que as apresentadas pelas famílias do estado e da região.

Conforme afirma Panigassi et al. ${ }^{50}$, a insegurança alimentar é um importante indicador para monitorar iniquidade, identificando grupos de vulnerabilidade social. Manifestam-se, assim, as carências e as privações sociais severas das famílias estudadas, possivelmente relacionadas com a vulnerabilidade do grupo.

Outro resultado importante deste estudo foi a expressiva prevalência de insegurança alimentar leve entre as crianças com déficit de estatura. Em estudo realizado por Pimentel et al. ${ }^{15}$ foi observada a associação entre insegurança alimentar e índices antropométricos, uma vez que crianças pertencentes às famílias com algum nível de insegurança alimentar apresentaram maior déficit ponderal e de estatura.

Por fim, cabe ressaltar que o desenho epidemiológico do tipo transversal traz limitações importantes, sendo uma delas a impossibilidade de analisar uma relação de causa e efeito entre as variáveis estudadas. Desta forma, os resultados observados não devem ser entendidos como fatores de risco para a desnutrição e excesso de peso e sim como associações entre eventos, em função da transversalidade do estudo ${ }^{51}$. Estudos longitudinais poderiam elucidar os fatores causais dos distúrbios nutricionais na infância, bem como avaliar a interferência das creches de João Pessoa sobre o estado nutricional dos seus usuários em idade pré-escolar e segurança alimentar de suas famílias.

\section{Conclusões}

O déficit de estatura e o excesso de peso foram os principais desvios antropométricos observados em crianças pré-escolares assistidas em creches públicas estaduais localizadas no município de João Pessoa, Paraíba. O esquema vacinal incompleto foi determinante proximal associado à forma crônica de desnutrição infantil, constituindo importante condição que deve ser considerada nas políticas públicas atuais. O perfil nutricional das crianças e a situação de segurança alimentar e nutricional das famílias convergem para a necessidade de melhorar a qualidade dos cuidados oferecidos, a fim de tornar as creches um eficiente e efetivo aparelho na prevenção de distúrbios nutricionais e na promoção de segurança alimentar e nutricional. 


\section{Colaboradores}

MM Souza participou da análise e interpretação dos dados, redação e correção final do artigo, da sua revisão crítica e da aprovação da versão a ser publicada; D Figueroa Pedraza participou da con- cepção e do delineamento do artigo, da sua correção final, da revisão crítica e da aprovação da versão a ser publicada; TN Menezes participou da redação e da correção final do artigo, da sua revisão crítica e da aprovação da versão a ser publicada.

\section{Referências}

1. Monteiro CA, Conde WL. Tendência secular da desnutrição e da obesidade na infância na cidade de São Paulo (1974-1996). Rev Saude Publica 2000; 34(6):52-61.

2. Conde WL, Gigante DP. Epidemiologia da desnutrição infantil. In: Kac G, Sichieri R, Gigante DP. Epidemiologia nutricional. Rio de Janeiro: Fiocruz, Atheneu; 2007. p. 281-295.

3. Silva MV, Sturion GL. Frequência à creche e outros condicionantes do estado nutricional infantil. Rev Nutr 1998; 11(1):58-68.

4. Romani SAM, Lira PIC. Fatores determinantes do crescimento infantil. Rev Bras Saúde Matern Infant 2004; 4(11):15-23.

5. Silva MV, Ometto AMH, Furtuoso MCO, Pipitone MAP, Sturion GL. Acesso à creche e estado nutricional das crianças brasileiras: diferenças regionais, por faixa etária e classe de renda. Rev Nutr 2000; 13(3):193-139.

6. Gibson RS, Hotz C. Nutritional causes of linear growth faltering in infants during the complementary feeding period. In: Martorell $\mathrm{R}$, Haschke F, editors. $\mathrm{Nu}$ trition and Growth. Nestle Nutrition Workshop Series Pediatric Program V.47. Philadelphia: Lippincott, Williams \& Wilkins; 2001. p. 159-192.

7. Neufeld L, Hotz C, para el Proyecto Challenges for Childhood Health and Nutrition Research in Latin America: addressing the 90/10 gap. Restricción en el crecimiento y deficiencia de micronutrientes. Bangladesh: Child Health and Nutrition Research Initiative; 2003.

8. Centro Brasileiro de Análise e Planejamento (CEBRAP), Ministério da Saúde (MS). Pesquisa Nacional Sobre Demografia e Saúde da criança e da mulher. Brasil: CEBRAP, MS; 2008.

9. Batista Filho M, Rissin A. A transição nutricional no Brasil: tendências regionais e temporais. Cad Saude Publica 2003; 19(Supl. 1):181-191.

10. Segall-Corrêa AM, Gonçalves NNS, Chalita LVAS, Russo-Leite GP, Padovani CR, Gonçalves A. Determinantes da evolução do peso e altura em crianças de 3 meses a 6 anos assistidas em creche: análise por modelo linear não hierarquizado em ensaio quaseexperimental. Rev Panam Salud Publica 2002; 12(1):19-25.

11. Coutinho JG, Gentil PC, Toral N. A desnutrição e obesidade no Brasil: o enfrentamento com base na agenda única da nutrição Cad Saude Publica 2008; 24(Supl. 2):332-340.
12. Zöllner CC, Fisberg RM. Estado nutricional e sua relação com fatores biológicos, sociais e demográficos de crianças assistidas por creches da Prefeitura do Município de São Paulo. Rev Bras Saúde Matern Infant 2006; 6(3):319-328.

13. Maluf RSJ, Menezes F, Marques SB. Caderno 'Segurança Alimentar', 2000. [texto da internet]. 2000 [acessado 2011 abr 20]. Disponível em: http://www. forumsocialmundial.org.br/download/tconferencias _Maluf_Menezes_2000_por.pdf

14. Brasil. II Conferência Nacional de Segurança Alimentar e Nutricional: A construção da Política Nacional de Segurança Alimentar. Relatório final- 2004. [texto da internet] 2004. [acessado 2007 mai 17]. Disponível em: http://www.fomezero.gov.br

15. Pimentel PG, Sichieri R, Salles-Costa R. Insegurança alimentar, condições socioeconômicas, indicadores antropométricos em crianças da região metropolitana do Rio de Janeiro/Brasil. R Bras Est Pop 2009; 26(2):283-294.

16. World Health Organization (WHO). Physical Status: The use and interpretation of anthropometry. Report of a WHO Expert Committee. Geneva: WHO; 1995. (Who Technical Report Series, n. 854)

17. de Onis M, Onyango AW, Van den Broeck J, Chumlea WC, Martorell R, for the WHO Multicentre Growth Reference Study Group. Measurement and standardization protocols for anthropometry used in the construction of a new international growth reference. Food Nutr Bull 2004; 25(Supl. 1):15-27.

18. World Health Organization (WHO). WHO global database on child growth and malnutrition. Geneva: WHO; 2007. [acessado 2010 mar 25]. Disponível em: http://www.who.int/nutgrowthdb/index.html

19. National Center for Health Statistics, Centers for Disease Control and Prevention. National Health and Nutrition Examination Survey. USA: CDC; 2000. [acessado 2010 abr 1]. Disponível em: http:// www.cdc.gov/growthcharts

20. Victora CG, Huttly SR, Fuchs SC, Olinto MT. The role of conceptual frameworks in epidemiological analysis: a hierarquical approach. Int J Epidemiol 1997; 26(1):224-227. 
21. Segall-Corrêa AM, Pérez-Escamilla R, Maranha LK, Sampaio MFA, Yuyana L, Alencar F, Vianna RPT, Vieira ACF, Coitinho D, Schmitz BS, Leão MM, Gubert M, Albuquerque ZP. Projeto: acompanhamento e avaliação da segurança alimentar em famílias brasileiras: validação de metodologia e de instrumento de coleto de informação. Campinas: Departamento de Medicina Preventiva e Social, Universidade Estadual de Campinas. Organização Pan-Americana da Saúde. Ministério da Saúde; 2003. (Relatório Técnico).

22. Maletta H, Gómez R. Seguridad Alimentaria: medición y métodos. Roma: FAO; 2004.

23. Hosmer DW, Lemeshow S, editors. Applied Logistic Regresssion. $2^{\text {nd }}$ Édition. New York: John Wiley \& Sons, INC; 2000.

24. Corso ACT, Viteritte PL, Peres MA. Prevalência de sobrepeso e sua associação com a área de residência em crianças menores de 6 anos de idade matriculadas em creches públicas de Florianópolis, Santa Catarina, Brasil. Rev Bras Epidemiol 2004; 7(2):201-209.

25. Fisberg RM, Marchioni DML, Cardoso MRA. Estado nutricional e fatores associados ao déficit de crescimento de crianças frequentadoras de creches públicas do município de São Paulo, Brasil. Cad Saude Publica 2004; 20(3):812-817.

26. Silva MV. A freqüência à creche influencia o estado nutricional infantil? Nutrire 2004; 27:1-17.

27. de Onis M, Frongillo EA, Blössner N. Is malnutrition declining? An analysis of changes in levels of child malnutrition since 1980. Bull World Health Organ 2000; 78(10):1222-1233.

28. Brasil. Ministério do Desenvolvimento Social e Combate a Fome (MDS). Secretaria de Avaliação e Gestão da Informação, editores. Chamada nutricional Quilombola 2006: Resumo Executivo. Brasília: MDS; 2007.

29. Brasil. Ministério da Saúde (MS). Associação Brasileira de Pós-Graduação em Saúde Coletiva, Institute of Ibero-American Studies. Inquérito Nacional de Saúde e Nutrição dos Povos Indígenas: Relatório Final (Análise dos Dados). Rio de Janeiro: MS; 2009.

30. Monte CMG. Desnutrição: um desafio secular à nutrição infantil. J Pediat 2000; 76(Supl. 3):285-297.

31. Souza CPC, Souza MPC, Rocha ACD, Figueroa Pedraza D. Perfil epidemiológico do estado nutricional de crianças assistidas em creches no estado da Paraíba. Nutrire 2011; 36(1):111-126.

32. Martins IS, Marinho SP, Oliveira DC, Araújo EAC. Pobreza, desnutrição e obesidade: inter-relação de estados nutricionais de indivíduos de uma mesma família. Cien Saude Colet 2007; 12(6):1553-1565.

33. Engstrom EM, Anjos LA. Déficit estatural nas crianças brasileiras: relação com condições sócio-ambientais e estado nutricional materno. Cad Saude Publica 1999; 15(3):559-567.

34. Oliveira LPM, Barreto ML, Assis AMO, Braga-Junior ACR, Nunes MFFP, Oliveira NF, Venâncio SI, Sladiva SRDM, Escuder MML. Preditores do retardo de crescimento linear em pré-escolares: uma abordagem multinível. Cad Saude Publica 2007; 23(3):601-613.

35. Fundo das Nações Unidas para a Infância (UNICEF). The State of the world's children 1998. Oxford: United Nations Children's Fund, Oxford University Press; 1997.
36. Brasil. Ministério da Saúde (MS). Secretaria de Atenção à Saúde. Departamento de Atenção Básica. Saúde da criança: nutrição infantil: aleitamento materno e alimentação complementar. Brasília: MS; 2009.

37. Bueno MB, Fisberg RM. Comparação de três critérios de classificação de sobrepeso e obesidade entre préescolares. Rev Bras Matern Infant 2006; 6(4):411-417.

38. Tuma RCFB, Costa THM, Schimitz BAS. Avaliação antropométrica e dietética de pré-escolares em três creches de Brasília, Distrito Federal. Rev Bras Saúde Matern Infant 2005; 5(4):419-428.

39. Corso ACT, Viteritte PL, Peres MA. Prevalência de sobrepeso e sua associação com a área de residência em crianças menores de 6 anos de idade matriculadas em creches públicas de Florianópolis, Santa Catarina, Brasil. Rev Bras Epidemiol 2004; 7(2):201-209.

40. NSW Centre for Public Health Nutrition. Best options for promoting healthy weight and preventing weight gain in NSW. New South Wales: University of Sidney; 2005.

41. Gill TP. Key issues in the prevention of obesity. $\mathrm{Br}$ Med Bull 1997; 53(2):359-388.

42. Dietz WH, Gortmaker SL. Preventing obesity in children and adolescents. Annu Rev Public Health 2001; 22:337-353.

43. Skinner JD, Carruth BR, Bounds W, Ziegler PJ. Children's food preferences: a longitudinal analysis. J Am Diet Assoc 2002; 102(11):1638-1647.

44. Gonzáles GJ, Vega MG. Condiciones sociodemograficas y estado nutricional de niños mesnores de un año em areas perifericas de Guadalajara, México. Rev Saúde Pública 1994; 28(4):268-276.

45. Sichieri R, Taddei JA, Everhart JE. Influence of parental height and sociodemographic factors on adolescent height in Brazil. J Adolesc Health 2000; 26(6):414-419.

46. Margarey AM, Daniels LA, Boulton TJ, Cockington RA. Predicting obesity in early adulthood from childhood and parenteral obesity. Int J Obes Relat Metab Disord 2003; 27(4): 505-513.

47. Martins EB, Carvalho MS. Associação entre o peso ao nascer e o excesso de peso na infância: revisão sistemática. Cad Saude Publica 2006; 22(11):2281-2300.

48. Vitolo MR, Gama CM, Bortolini GA, Campagnolo PDB, Drachler ML. Some risk factors associated with overweight, stunting and wasting among children under 5 years old. I Pediatr 2008; 84(3):251-257.

49. Instituto Brasileiro de Geografia e Estatística (IBGE). Pesquisa Nacional por Amostra de Domicílios: segurança alimentar 2004/2009. Rio de Janeiro: IBGE; 2010.

50. Panigassi G, Segall-Corrêa AM, Marin Leon L, Pérez-Escamilla R, Sampaio MFA, Maranha LK. Insegurança alimentar como indicador de iniquidade: análise de inquérito populacional. Cad Saude $\mathrm{Pu}$ blica 2008; 24(10):2376-2384.

51. Barroso GS, Sichieri R, Salles-Costa R. Fatores associados ao déficit nutricional em crianças residentes em uma área de prevalência elevada de insegurança alimentar. Rev Bras Epidemiol 2008; 11(3): 484-494.

Artigo apresentado em 21/08/2011

Aprovado em 14/10/2011

Versão final apresentada em 19/12/2011 